\title{
Evaluation of Firm's Loss Due to Incomplete Information in Real Investment Decision
}

\author{
Michi NISHIHARA $^{a}$, Masao FUKUSHIMA $^{a 1}$ \\ ${ }^{a}$ Department of Applied Mathematics and Physics, Graduate School of Informatics, \\ Kyoto University, Kyoto 6068175, Japan.
}

February 9, 2006; revised January 31, 2007; March 20, 2007

\begin{abstract}
We investigate the effect of incomplete information in a model where a start-up with a unique idea and technology pioneers a new market but will eventually be expelled from the market by a large firm's subsequent entry. We evaluate the startup's loss due to incomplete information about the large firm's behavior. We clarify conditions under which the start-up needs more information about the large firm. The proposed method of evaluating the loss due to incomplete information could also be applied to other real options models involving incomplete information. Keywords: Applied probability; Investment analysis; Real options; Incomplete information; Leader-follower game
\end{abstract}

\section{Introduction}

The real options approach has become an increasingly standard framework for investment timing decisions. In the real options approach, a firm that faces an irreversible investment generating uncertain profit in future is considered to have an option to make the investment. Then, in order to maximize the expected profit, the firm must invest when the NPV (net present value) of the investment becomes greater than the opportunity cost of investing (i.e., the value of the option to delay the investment). The real options

\footnotetext{
${ }^{1}$ Corresponding author. Tel: +81 75753 4756; fax: +81 75753 4756. E-mail addresses: fuku@i.kyotou.ac.jp (M. Fukushima), michi@amp.i.kyoto-u.ac.jp (M. Nishihara).
} 
approach to investment has provided a new insight into a firm's real investment decision which tends to rely on managerial experiences and intuitions, and it has gradually come to be applied to investment in the real world since the proposal by Dixit (1989) and McDonald and Siegel (1986).

Although the early literature considered the investment decision of monopolists, more recent studies (see Boyer, Gravel, and Lasserre (2004) for an overview) have investigated how the firm's investment decision is affected by its rival firms' behaviors because an opportunity to make investment, unlike financial options, can usually be shared by several firms in the same industry. One of the earliest results in the strategic real options has been obtained by Grenadier (2002), who studied the symmetric equilibrium strategy for firms. Weeds (2002) has derived equilibrium strategies in two players who attempt to preempt a single patent from the other, and Huisman and Kort (2003) have investigated a two player real options game in the context of the adoption of new technology.

While the above studies assume complete information about the competitors, Lambrecht and Perraudin (2003) consider a model involving incomplete information about the competitors' investment costs. Hsu and Lambrecht (2003) introduce asymmetric and incomplete information in real options in the context of a patent race. Using the filtering theory, Bernardo and Chowdhry (2002) and Décamps, Mariotti, and Villeneuve (2005) have investigated models in which a firm has incomplete information about parameters of its own profit flow rather than the competitors' behavior. Furthermore, Grenadier and Wang (2005) and Nishihara and Shibata (2007) have examined the effect of asymmetric information between the owner and the manager in the single firm.

The effect of incomplete information is practically significant, since how accurately a firm can estimate the behaviors of rival firms has a crucial effect on whether or not its investment succeeds. The previous studies such as Lambrecht and Perraudin (2003) and Hsu and Lambrecht (2003) derived the values and the optimal strategies under incomplete information simultaneously. However, in their approach, the value under incomplete information has an element of the firm's estimation and hence it may exceed the value under complete information. In order to reveal how great loss a firm may suffer due to incomplete information, we examine the value of the project from a different aspect. Actually, we regard the value derived simultaneously with the optimal stopping time under incom- 
plete information as what the firm believes. We, unlike the previous studies, calculate the real expected payoff, which is different from the value that the firm believes. Then, we derive the loss due to incomplete information as the difference between the real expected payoff and the expected payoff in the case of complete information. This analysis is useful to unveil a risk of a firm using the real options approach under incomplete information.

This paper examines a model with a start-up who pioneers a new market by a unique idea and technology and a large firm that will eventually take over the market from the start-up. We evaluate the start-up's loss due to incomplete information about the large firm. Then, we clarify conditions under which the start-up needs more information about the large firm. Moreover, we show that in some cases the real options strategy under incomplete information gives less expected payoff to the start-up than the zeroNPV strategy (i.e., investing when the NPV of the investment becomes positive) under the same incomplete information. Our results suggest that in some cases a firm using the real options approach to investment has a great risk of incorrect conjectures about the behaviors of its competitors. Although we consider the simple model involving two firms for the purpose of concentrating our attention on the loss due to incomplete information, the proposed method of evaluating the loss due to incomplete information could also be applied to other real options models involving several firms.

This paper is organized as follows. After the model is introduced in Section 2, Section 3 derives the start-up's value function and optimal strategy under complete information. Section 4 describes our main theoretical results, which show the start-up's strategy under incomplete information, its real expected payoff, and the loss due to incomplete information. In Section 5, we discuss how similar results can be obtained in a general situation, although the analysis in Sections 3 and 4 limit attention to a simple situation for mathematical convenience. Section 6 provides several implications with numerical examples. Section 7 concludes the paper.

\section{Model}

This section introduces the model treated in this paper. We consider the start-up (leader)'s problem of determining the timing of entering the new market which may be taken over 
by the large firm (follower) eventually. In this problem, we will discuss how incomplete information about the large firm affects the expected payoff of the start-up. Throughout this paper, we assume that both stochastic process and random variable are defined on the filtered probability space $\left(\Omega, \mathcal{F}, P ; \mathcal{F}_{t}\right)$. The model is described as follows:

Profit flows and investment costs of the two firms: The start-up can receive a profit flow $D_{1}(1,0) Y(t)$ in the new market by paying an indivisible investment cost $I_{1}$, but the flow will be reduced to $D_{1}(1,1) Y(t)$ after the large firm's entry to the market. Here, $(1,0)$ (resp. $(1,1)$ ) denotes the situation in which only the start-up (resp. both firms) is active in the market. Quantities $I_{1}, D_{1}(1,1)$ and $D_{1}(1,0)$ are constants such that $I_{1}>0$ and $0 \leq D_{1}(1,1)<D_{1}(1,0)$, and $Y(t)$ is the market demand satisfying the following geometric Brownian motion:

$$
\mathrm{d} Y(t)=\mu Y(t) \mathrm{d} t+\sigma Y(t) \mathrm{d} B(t) \quad(t>0), \quad Y(0)=y
$$

where $\mu(\geq 0), \sigma(>0)$ and $y(>0)$ are given constants, and $B(t)$ denotes the onedimensional $\mathcal{F}_{t}$ standard Brownian motion. In contrast, the large firm does not notice the existence of the potential market until the start-up's investment. The large firm can obtain a profit flow $D_{2}(1,1) Y(t)$ in the market by paying an indivisible investment cost $I_{2}$ after the start-up's investment. Here, $I_{2}$ and $D_{2}(1,1)$ are positive constants. The adapted process $Y(t)$ captures observable (and exogenous) market demand at time $t$, while $D_{i}(\cdot, \cdot)(i=1,2)$ captures the endogenous change in firm cash flows resulting from the respective firms' entrance in the market.

The large firm's investment decision: The large firm does not notice the opportunity to preempt the market until the date $\tau_{1}$ on which the start-up invests. Then, with discount rate $\rho(>\mu)$, the large firm optimizes its investment time $\tau_{2}$ by solving the following optimal stopping problem:

$$
\sup _{\tau_{2} \geq \tau_{1}} E\left[\int_{\tau_{2}}^{\infty} \mathrm{e}^{-\rho t} D_{2}(1,1) Y(t) \mathrm{d} t-\mathrm{e}^{-\rho \tau_{2}} I_{2}\right],
$$

where $\tau_{2}$ is any $\mathcal{F}_{t}$ stopping time that satisfies $\tau_{2} \geq \tau_{1}$. Let us call $Q_{1}=D_{1}(1,0) / I_{1}$ and $Q_{2}=D_{2}(1,1) / I_{2}$ the efficiencies of the start-up's and the large firm's investment, respectively. The efficiencies will be influenced by the profit margin in addition to the firm's idea and technology standard. This paper considers a situation in which the large firm sets a smaller profit margin in order to take over the market from the leader. For 
that reason, the large firm's efficiency is likely to be lower than that of the start-up. Let $\tau_{2}^{q}$ denote the optimal stopping time of problem (2) with $Q_{2}=D_{2}(1,1) / I_{2}$ replaced by a general constant $q(>0)$.

The start-up's investment decision: Since the start-up does not have complete information about the efficiency of the large firm, the start-up determines its investment time $\tau_{1}$ assuming that the efficiency of the large firm obeys a random variable $X$ independent of filtration $\left\{\mathcal{F}_{t}\right\}$. Then, the start-up believes that its expected payoff of investing at $\tau_{1}$ is equal to

$$
E\left[\int_{\tau_{1}}^{\tau_{2}^{X}} \mathrm{e}^{-\rho t} D_{1}(1,0) Y(t) \mathrm{d} t+\int_{\tau_{2}^{X}}^{+\infty} \mathrm{e}^{-\rho t} D_{1}(1,1) Y(t) \mathrm{d} t-\mathrm{e}^{-\rho \tau_{1}} I_{1}\right],
$$

where $\tau_{2}^{X}$ represents a random variable which takes a value $\tau_{2}^{X(\omega)}(\omega)$ for $\omega \in \Omega$ (note that $\tau_{2}^{X}$ also depends on $\tau_{1}$ ). The start-up finds its investment time $\tau_{1}$ by solving the following optimal stopping problem:

$$
V(y)=\sup _{\tau_{1}} E\left[\int_{\tau_{1}}^{\tau_{2}^{X}} \mathrm{e}^{-\rho t} D_{1}(1,0) Y(t) \mathrm{d} t+\int_{\tau_{2}^{X}}^{+\infty} \mathrm{e}^{-\rho t} D_{1}(1,1) Y(t) \mathrm{d} t-\mathrm{e}^{-\rho \tau_{1}} I_{1}\right],
$$

where $\tau_{1}$ is any $\mathcal{F}_{t}$ stopping time. Let $V(y)$ (recall $y=Y(0)$ ) and $\tau_{1}^{*}$ denote the value function and the optimal stopping time in problem (4), respectively. The optimal stopping time $\tau_{1}^{*}$ is expressed in a form independent of the initial value $y$, as will be shown in Sections 3 and 4 . Let $V(y ; q)$ and $\tau_{1}^{q}$ be the value function and the optimal stopping time, respectively, in problem (4) with $X$ replaced by a constant $q(>0)$. Note that, if the start-up has the complete information on the large firm's efficiency $Q_{2}$, the start-up invests at $\tau_{1}^{Q_{2}}$ and its real expected payoff agrees with $V\left(y ; Q_{2}\right)$.

Remark 2.1 For simplicity, this paper treats the two player leader-follower game as mentioned above. Similar results can be obtained in a more practical setting that permits several followers, by assuming that the followers make joint investment. There is a possibility that the followers make joint investment even if they are non-cooperative. For details, see Huisman (2001).

Dixit and Pindyck (1994) and Huisman (2001) have investigated a preemption model in which both firms attempt to become a leader assuming complete information. Unlike their model, the model studied in this paper is a leader-follower game. Indeed, we model 
a situation where a small entrepreneurial firm has the advantage of pioneering a new market, while a large follower has a big power of taking over the market from the small leader. In Sections 3 and 4 , we assume $0=D_{1}(1,1)<D_{2}(1,1)$ to avoid mathematical clutter and understand the essence of the loss due to incomplete information. Since this assumption is extreme, we consider a more realistic setting, i.e., $D_{1}(1,1)>0$ in Section 5 and explain how similar results are obtained. In the rest of the paper, we will denote for simplicity $D_{1}=D_{1}(1,0)$ and $D_{2}=D_{2}(1,1)$ unless they cause confusion.

\section{Case of complete information}

This section derives the value function $V(y ; q)$ and the optimal stopping time $\tau_{1}^{q}$ of the start-up who believes that the efficiency of the large firm's investment is a constant $q(>0)$. That is, we consider problem (4) with $X \equiv q$. As in Dixit and Pindyck (1994) and Huisman (2001), we solve the leader-follower game backwards.

First, we begin by supposing that the start-up (leader) has already invested at time $\tau_{1}$, and derive the optimal stopping time $\tau_{2}^{q}$ of the large firm (follower). That is, we consider the follower's problem. Under the assumption that the start-up has already entered the market, the large firm's problem (2) can be treated as a problem for a monopolist. Therefore, the large firm's optimal stopping time $\tau_{2}^{q}$ in problem (2) with $Q_{2}=q$ is expressed as follows:

$$
\tau_{2}^{q}=\inf \left\{t \geq \tau_{1} \mid Y(t) \geq y_{M}(q)\right\}
$$

where we define

$$
\begin{aligned}
y_{M}(q) & =\frac{\beta_{1}(\rho-\mu)}{\left(\beta_{1}-1\right) q} \quad(q>0), \\
\beta_{1} & =\frac{1}{2}-\frac{\mu}{\sigma^{2}}+\sqrt{\left(\frac{\mu}{\sigma^{2}}-\frac{1}{2}\right)^{2}+\frac{2 \rho}{\sigma^{2}}} \\
\beta_{2} & =\frac{1}{2}-\frac{\mu}{\sigma^{2}}-\sqrt{\left(\frac{\mu}{\sigma^{2}}-\frac{1}{2}\right)^{2}+\frac{2 \rho}{\sigma^{2}}} .
\end{aligned}
$$

Here, $\beta_{i}(i=1,2)$ are usual characteristic roots in an optimal stopping problem with discount rate $\rho$ and state process $Y(t)$ following (1), and we can easily check $\beta_{1}>1$ and $\beta_{2}<0$ by $\sigma>0$ and $0 \leq \mu<\rho$. The function $y_{M}(q)$ represents the optimal investment trigger of a monopolist with efficiency $q$ (see, for example, Dixit and Pindyck (1994)). 
Next, using the large firm's response $\tau_{2}^{q}$ derived as (5), we calculate the start-up's value function $V(y ; q)$ and investment time $\tau_{1}^{q}$ in problem (4) with $X \equiv q$. That is, we consider the leader's problem. Before stating the proposition, we define the function

$$
p\left(\beta_{1}, Q_{1}, q\right)=\left(\frac{1}{\beta_{1}}\right)^{\frac{1}{\beta_{1}-1}}-\frac{q}{Q_{1}} \quad\left(\beta_{1}>1, Q_{1}>0, q>0\right),
$$

which values how large the start-up's efficiency $Q_{1}$ is against $q$. The importance of the function $p\left(\beta_{1}, Q_{1}, q\right)$ will be mentioned after the following proposition.

Proposition 3.1 The start-up's value function $V(y ; q)$ and optimal stopping time $\tau_{1}^{q}$ are given as follows. If

$$
p\left(\beta_{1}, Q_{1}, q\right)>0
$$

then

$$
V(y ; q)= \begin{cases}A(q) y^{\beta_{1}} & \left(0<y<y_{M}\left(Q_{1}\right)\right) \\ \frac{D_{1} y}{\rho-\mu}-I_{1}-\frac{D_{1} y_{M}(q)^{-\beta_{1}+1} y^{\beta_{1}}}{\rho-\mu} & \left(y_{M}\left(Q_{1}\right) \leq y \leq y_{U}(q)\right) \\ B(q) y^{\beta_{2}} & \left(y>y_{U}(q)\right),\end{cases}
$$

and $\tau_{1}^{q}$ is expressed as $\tau_{1}^{q}=\inf \left\{t \geq 0 \mid Y(t) \in\left[y_{M}\left(Q_{1}\right), y_{U}(q)\right]\right\}$ (i.e., a hitting time into the interval $\left.\left[y_{M}\left(Q_{1}\right), y_{U}(q)\right]\right)$ regardless of the initial value $Y(0)=y$. Here, $y_{M}\left(Q_{1}\right)$ is the threshold defined by (6) with $q=Q_{1}$, and $A(q)$ is defined by

$$
A(q)=y_{M}\left(Q_{1}\right)^{-\beta_{1}}\left(\frac{D_{1} y_{M}\left(Q_{1}\right)}{\rho-\mu}-I_{1}-\frac{D_{1} y_{M}(q)^{-\beta_{1}+1} y_{M}\left(Q_{1}\right)^{\beta_{1}}}{\rho-\mu}\right) \quad(q>0) .
$$

Moreover, for $q>0$ satisfying $(10), y_{U}(q)$ is the threshold defined by the unique solution of the equation

$$
\frac{\left(\beta_{1}-\beta_{2}\right) Q_{1} y_{M}(q)^{-\beta_{1}+1}}{\rho-\mu} y^{\beta_{1}}+\frac{\left(\beta_{2}-1\right) Q_{1}}{\rho-\mu} y-\beta_{2}=0 \quad\left(y_{M}\left(Q_{1}\right)<y<y_{M}(q)\right),
$$

and $B(q)$ is defined by

$$
B(q)=y_{U}(q)^{-\beta_{2}}\left(\frac{D_{1} y_{U}(q)}{\rho-\mu}-I_{1}-\frac{D_{1} y_{M}(q)^{-\beta_{1}+1} y_{U}(q)^{\beta_{1}}}{\rho-\mu}\right) .
$$

If (10) does not hold, then $V(y ; q)=0$ for all $y>0$ and $\tau_{1}^{q}=+\infty$.

(Proof) See Appendix A. 
Remark 3.1 Until the large firm's efficiency $q$ exceeds the solution of $p\left(\beta_{1}, Q_{1}, q\right)=$ 0 , inequality $(10)$ holds, and $y_{U}(q)$ and $V(y ; q)$ monotonically decrease with $q$. On the contrary, we have $y_{U}(q) \uparrow+\infty$ and $\tau_{1}^{q} \rightarrow \inf \left\{t \geq 0 \mid Y(t) \geq y_{M}\left(Q_{1}\right)\right\}$ as $q \downarrow 0$; this means that the stopping time $\tau_{1}^{q}$ tends to the optimal stopping time of a monopolist.

Remark 3.2 By taking $q=Q_{2}$ in Proposition 3.1, we obtain the real expected payoff $V\left(y ; Q_{2}\right)$ of the start-up who has complete information about the efficiency of the large firm.

We explain equation (11) in Proposition 3.1. The interval $\left[y_{M}\left(Q_{1}\right), y_{U}(q)\right]$ represents the stopping (investment) region, where the start-up immediately invests. The value function $V(y ; q)$ in this region consists of two components, namely the monopoly profit $D_{1} y /(\rho-\mu)-I_{1}$ and the subtracter $D_{1} y_{M}(q)^{-\beta_{1}+1} y^{\beta_{1}} /(\rho-\mu)$ which represents the effect of takeover by the follower. The remaining parts $\left(0, y_{M}\left(Q_{1}\right)\right)$ and $\left(y_{U}(q), \infty\right)$ represent the continuation region, where the start-up delays its investment until the market demand $Y(t)$ reaches one of the thresholds. However, the reasons why the start-up defers its investment in the two regions are completely different. When $Y(t)$ lies in the region $\left(0, y_{M}\left(Q_{1}\right)\right)$, the start-up waits until the market demand level $y_{M}\left(Q_{1}\right)$ is achieved so as to obtain a good profit from the investment. On the other hand, when $Y(t)$ is in the region $\left(y_{U}(q), \infty\right)$, the start-up waits for the market demand $Y(t)$ to fall down to $y_{U}(q)$ so as to prevent the follower from investing too early. Note that the start-up's investment trigger $y_{M}\left(Q_{1}\right)$ remains unchanged from that of the monopolist regardless of the large firm's efficiency $q$, as far as inequality (10) is satisfied. The value functions $A(q) y^{\beta_{1}}$ and $B(q) y^{\beta_{1}}$ in the continuation regions mean the values of the options to invest at the triggers $y_{M}\left(Q_{1}\right)$ and $y_{U}(q)$, respectively.

As the presence of the upper trigger $y_{U}(q)$ is a distinctive feature of our leader-follower model, we will explain it in more detail. Proposition 3.1 suggests that if the initial value $Y(0)=y$ is larger than $y_{U}(q)$, the start-up should delay its investment until the market demand $Y(t)$ drops to the threshold $y_{U}(q)$. Note that $Y(t)$ could decrease from the initial value $y$ to the threshold $y_{U}(q)$ even with a positive drift $\mu$ in (1) because of the positive volatility $\sigma$ in (1). Even if the start-up makes its investment in the case of $Y(t)>y_{U}(q)$, the large firm is quite likely to enter the market before the start-up gains a sufficient cash flow. Thus, the start-up defers its investment when the market demand is great. 
Inequality (10) can be interpreted as a prerequisite condition for the start-up's investment. In fact, the start-up's expected payoff never becomes positive for any time $t$ and any value of $Y(t)$, unless (10) holds (for details see Appendix A). Now we examine how the prerequisite condition (10) is changed by the values of parameters $\mu, \rho$ and $\sigma$. We can see from (7) that $\partial \beta_{1} / \partial \sigma<0, \lim _{\sigma \uparrow+\infty} \beta_{1}=1, \lim _{\sigma \downarrow 0} \beta_{1}=\rho / \mu>1, \partial \beta_{1} / \partial \mu<0$ and $\partial \beta_{1} / \partial \rho>0$ (see Dixit and Pindyck (1994)). Since $p\left(\beta_{1}, Q_{1}, q\right)$ is monotonically increasing for $\beta_{1}>1$ by (9), the prerequisite condition (10) becomes more restrictive, i.e., the opportunity for the start-up to invest is more likely to be lost, as the drift $\mu$ and the volatility $\sigma$ (resp. the discount rate $\rho$ ) in the market increase (resp. decrease). This is because the start-up's investment opportunity is greatly affected by the large firm. That is, increases in the drift and volatility raise the probability of the large firm's entry. Consequently, it is harder for the start-up to find the opportunity to obtain enough profits before the takeover by the big follower. Moreover, we have $p\left(\beta_{1}, Q_{1}, q\right) \downarrow 1 / \mathrm{e}-q / Q_{1}$ as $\beta_{1} \downarrow 1$, and $p\left(\beta_{1}, Q_{1}, q\right) \uparrow 1-q / Q_{1}$ as $\beta_{1} \uparrow+\infty$ by (9). Hence, if the start-up's efficiency $Q_{1}$ is e times larger than that of the large firm, the prerequisite condition (10) always holds and the start-up's entrepreneurial activity is absolutely valuable for any values of parameters $\mu, \rho$ and $\sigma$. If the start-up's efficiency $Q_{1}$ is less than that of the large firm, on the other hand, then the prerequisite condition never holds, which means that the start-up has no opportunity to make the entrepreneurial activity.

Finally, it should be noted that the complete information version has an element of incomplete information, because the large firm does not learn about the investment opportunity until the start-up makes its investment. The start-up knows this and uses its informational advantage in determining its optimal investment. The next section describes our main results, which evaluate the start-up's loss due to incomplete information about the efficiency of the large firm's investment

\section{Loss due to incomplete information}

This section evaluates the start-up's loss due to incomplete information about the efficiency of the large firm's investment by the following procedure:

Step 1: Derive the value function $V(y)$ and the optimal stopping time $\tau_{1}^{*}$ in problem (4) 
which the start-up believes.

Step 2: Calculate the real expected payoff $\tilde{V}(y)$ of the start-up who invests at time $\tau_{1}^{*}$ calculated in Step 1.

Step 3: Derive $W(y)=V\left(y ; Q_{2}\right)-\tilde{V}(y)$, which is the difference between the expected payoff of the start-up who invests at time $\tau_{1}^{Q_{2}}$ under complete information and that of the start-up who invests at (wrong) time $\tau_{1}^{*}$ due to incomplete information.

The quantity $W(y)$ calculated in Step 3 is regarded as the loss due to incomplete information. Most of the existing works concerning real options under incomplete information consider only Step 1, namely the optimal strategy and the value that the firm believes under incomplete information. We however consider the real payoff in Step 2 and then compare the real payoff (which is different from the value in Step 1) and the value under complete information in Step 3. In the above procedure, we examine the loss which the firm suffers due to incomplete information. The proposed method may also be applied to other real options models involving incomplete information. The loss due to incomplete information is identified as the value of information about the rival firm, and hence it tells us whether the firm should conduct a further survey on the rival firm or not. Subsections 4.1, 4.2, and 4.3 describe Steps 1, 2, and 3, respectively.

\subsection{Start-up's strategy under incomplete information}

The start-up determines its investment time, believing that the large firm's efficiency obeys a random variable $X$ independent of the filtration $\left\{\mathcal{F}_{t}\right\}$. We call the random variable $X$ the start-up's estimation of the large firm's efficiency. Here we assume that $X>0$ and $E\left[X^{\beta_{1}-1}\right]<+\infty$. We define the function $g(y)$ by

$$
g(y)=\frac{D_{1} y}{\rho-\mu}-I_{1}-\frac{D_{1} E\left[\max \left(y_{M}(X), y\right)^{-\beta_{1}+1}\right] y^{\beta_{1}}}{\rho-\mu} \quad(y>0) .
$$

It can be seen in the calculations in Appendix B that $g(y)$ is equal to the expectation (3) with $\tau_{1}=0$, namely the payoff (that the start-up believes) by the immediate investment. Recall that $y$ is the initial market demand $y=Y(0)$. Generally, it is hard to derive an explicit form of the value function and the optimal stopping time in problem (4). However, we can show that problem (4) is reducible to the problem with $q=\tilde{Q}_{2}$ in Section 3, where we define $\tilde{Q}_{2}=E\left[X^{\beta_{1}-1}\right]^{1 /\left(\beta_{1}-1\right)}$, provided that the following condition holds: 
Condition (a): The inequality $g(y) \leq V\left(y ; \tilde{Q}_{2}\right)$ holds for all $y>0$.

The quantity $\tilde{Q}_{2}$ features the start-up's strategy under incomplete information as will be shown in the following proposition. In relation to (15), we define

$$
h(y)=\frac{D_{1} y}{\rho-\mu}-I_{1}-\frac{D_{1} E\left[y_{M}(X)^{-\beta_{1}+1}\right] y^{\beta_{1}}}{\rho-\mu} \quad(y>0) .
$$

From the definitions of $g(y), h(y), \tilde{Q}_{2}$ and Proposition 3.1, it immediately follows that

$$
\begin{aligned}
& h(y) \leq g(y) \quad(y>0), \\
& h(y)=V\left(y ; \tilde{Q}_{2}\right) \quad\left(y_{M}\left(Q_{1}\right)<y<y_{U}\left(\tilde{Q}_{2}\right), p\left(\beta_{1}, Q_{1}, \tilde{Q}_{2}\right)>0\right) .
\end{aligned}
$$

Using this property, we can show the following proposition, which is the key to evaluating the loss due to incomplete information.

Proposition 4.1 Assume that Condition (a) holds. The value function $V(y)$ and the optimal stopping time $\tau_{1}^{*}$ in problem (4) which the start-up believes are given as $V(y)=$ $V\left(y ; \tilde{Q}_{2}\right)$ and $\tau_{1}^{*}=\tau_{1}^{\tilde{Q}_{2}}$ for all $y>0$, respectively, where $V\left(y ; \tilde{Q}_{2}\right)$ and $\tau_{1}^{q}$ are given in Proposition 3.1.

(Proof) See Appendix B.

Remark 4.1 Condition (a) is likely to hold when the support of $X$ is not very wide. In particular, we can easily show that Condition (a) always holds whenever $X$ is a constant.

Remark 4.2 Figure 1 illustrates the function $V(y)=V\left(y ; \tilde{Q}_{2}\right)$ together with the functions $g(y)$ and $h(y)$ under Condition (a). In particular, we observe that $V(y)=V\left(y ; \tilde{Q}_{2}\right)=$ $g(y)=h(y)$ holds for $y \in\left[y_{M}\left(Q_{1}\right), y_{U}\left(\tilde{Q}_{2}\right)\right]$.

Proposition 4.1 shows the value function and the optimal stopping time of the startup with estimation $X$. It should be noted that the value $V(y)$ is just the one believed by the start-up and is different from the real expected payoff of the investment, $\tilde{V}(y)$, which will be calculated in the next subsection. By Proposition 4.1, the start-up with the estimation $X$ takes the same strategy as that of the start-up with the constant estimation $\tilde{Q}_{2}$ under Condition (a). That is, the start-up's estimation of the large firm's efficiency is completely characterized by the single quantity $\tilde{Q}_{2}=E\left[X^{\beta_{1}-1}\right]^{1 /\left(\beta_{1}-1\right)}$ independently of its distribution. However, this is not always true in a general case without Condition (a). In the rest of the paper, we will restrict our attention to the case where Condition (a) is satisfied. 


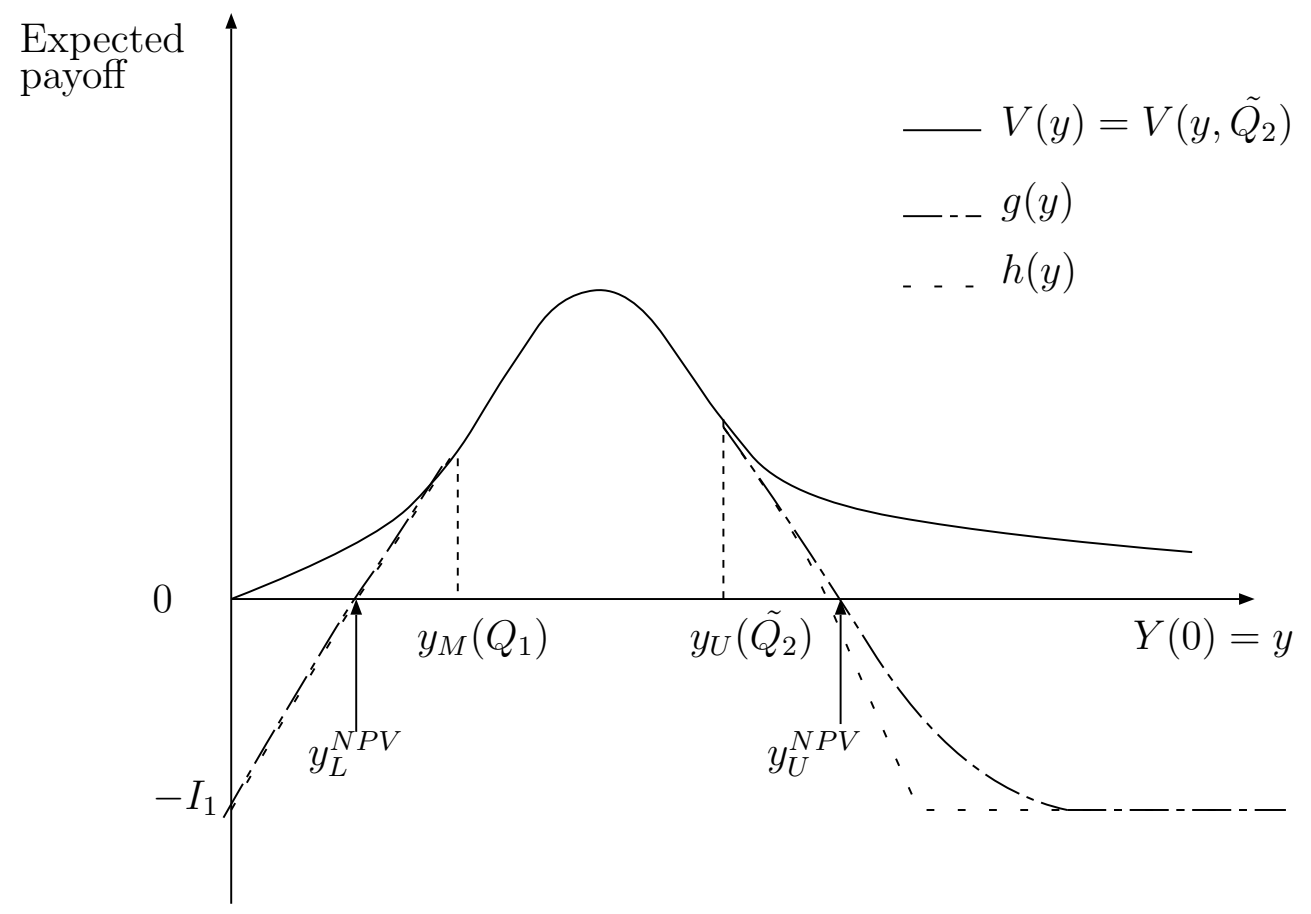

Figure 1: $g(y), h(y)$ and $V(y)=V\left(y ; \tilde{Q}_{2}\right)$.

\subsection{The real expected payoff of the start-up}

This subsection derives the real expected payoff $\tilde{V}(y)$ of the start-up who invests at time $\tau_{1}^{*}$ calculated in Proposition 4.1. Since the large firm's real efficiency is $Q_{2}$, its real investment time is equal to (5) with $q=Q_{2}$, i.e.,

$$
\tau_{2}^{Q_{2}}=\inf \left\{t \geq \tau_{1}^{*} \mid Y(t) \geq y_{M}\left(Q_{2}\right)\right\}
$$

Then, the start-up's real expected payoff $\tilde{V}(y)$ becomes

$$
\tilde{V}(y)=E\left[\int_{\tau_{1}^{*}}^{\tau_{2}^{Q_{2}}} \mathrm{e}^{-\rho t} D_{1} Y(t) \mathrm{d} t-\mathrm{e}^{-\rho \tau_{1}^{*}} I_{1}\right] .
$$

We can show the following proposition by calculating the expectation (20).

Proposition 4.2 Assume that Condition (a) holds. The real expected payoff $\tilde{V}(y)$ of the start-up who invests at $\tau_{1}^{*}$ is given as follows. If $p\left(\beta_{1}, Q_{1}, \tilde{Q}_{2}\right)>0$, then

$$
\tilde{V}(y)= \begin{cases}\tilde{A}\left(Q_{2}\right) y^{\beta_{1}} & \left(0<y<y_{M}\left(Q_{1}\right)\right) \\ \frac{D_{1} y}{\rho-\mu}-I_{1}-\frac{D_{1} \max \left(y, y_{M}\left(Q_{2}\right)\right)^{-\beta_{1}+1} y^{\beta_{1}}}{\rho-\mu} & \left(y_{M}\left(Q_{1}\right) \leq y \leq y_{U}\left(\tilde{Q}_{2}\right)\right) \\ \tilde{B}\left(\tilde{Q}_{2}\right) y^{\beta_{2}} & \left(y>y_{U}\left(\tilde{Q}_{2}\right)\right),\end{cases}
$$


where $y_{M}(\cdot)$ is defined by $(6), y_{U}\left(\tilde{Q}_{2}\right)$ is the unique solution of equation $(13)$ with $q=\tilde{Q}_{2}$, and $\tilde{A}\left(Q_{2}\right)$ and $\tilde{B}\left(\tilde{Q}_{2}\right)$ are defined by

$$
\begin{aligned}
& \left.\tilde{A}\left(Q_{2}\right)=y_{M}\left(Q_{1}\right)^{-\beta_{1}}\left(\frac{D_{1} y_{M}\left(Q_{1}\right)}{\rho-\mu}-I_{1}-\frac{D_{1} \max \left(y_{M}\left(Q_{1}\right), y_{M}\left(Q_{2}\right)\right)^{-\beta_{1}+1} y_{M}\left(Q_{1}\right)^{\beta_{1}}}{\rho-\mu}\right) 22\right) \\
& \tilde{B}\left(\tilde{Q}_{2}\right)=y_{U}\left(\tilde{Q}_{2}\right)^{-\beta_{2}}\left(\frac{D_{1} y_{U}\left(\tilde{Q}_{2}\right)}{\rho-\mu}-I_{1}-\frac{D_{1} \max \left(y_{U}\left(\tilde{Q}_{2}\right), y_{M}\left(Q_{2}\right)\right)^{-\beta_{1}+1} y_{U}\left(\tilde{Q}_{2}\right)^{\beta_{1}}}{\rho-\mu}\right)(.23)
\end{aligned}
$$

If $p\left(\beta_{1}, Q_{1}, \tilde{Q}_{2}\right) \leq 0$, then $\tilde{V}(y)=0$ for all $y>0$.

(Proof) See Appendix C.

Remark 4.3 Propositions 3.1, 4.1 and 4.2 ensure that $\tilde{V}(y)=V\left(y ; Q_{2}\right)=V\left(y ; \tilde{Q}_{2}\right)=$ $V(y)$ under Condition (a), whenever $\tilde{Q}_{2}=Q_{2}$.

We make a brief explanation about Proposition 4.2. If $p\left(\beta_{1}, Q_{1}, \tilde{Q}_{2}\right)>0$, then the start-up invests as soon as the market demand $Y(t)$ reaches the investment region $\left[y_{M}\left(Q_{1}\right), y_{U}\left(\tilde{Q}_{2}\right)\right]$. Then it obtains the expected cash flow (21), but (21) may be negative if the start-up's estimation of the large firm's efficiency is far from correct. Otherwise, the start-up makes a decision of never investing because it considers no value of the project due to the presence of the big follower.

\subsection{The start-up's loss due to incomplete information}

We evaluate the start-up's loss $W(y)=V\left(y ; Q_{2}\right)-\tilde{V}(y)$ due to incomplete information about the large firm's efficiency. The loss $W(y)$ varies according to the relation between $\tilde{Q}_{2}$ and $Q_{2}$. Note that $y_{M}(\cdot)$ is monotonically decreasing by definition (6).

Case $(\mathbf{U}): \tilde{Q}_{2}<Q_{2}$ The start-up underestimates the large firm's efficiency, and $y_{M}\left(\tilde{Q}_{2}\right)>$ $y_{M}\left(Q_{2}\right)$ holds with respect to the large firm's entry trigger.

Case (C): $\tilde{Q}_{2}=Q_{2}$ The start-up correctly estimates the large firm's efficiency, and $y_{M}\left(\tilde{Q}_{2}\right)=y_{M}\left(Q_{2}\right)$ holds with respect to the large firm's entry trigger.

Case $(\mathbf{O}): \tilde{Q}_{2}>Q_{2}$ The start-up overestimates the large firm's efficiency, and $y_{M}\left(\tilde{Q}_{2}\right)<$ $y_{M}\left(Q_{2}\right)$ holds with respect to the large firm's entry trigger.

Proposition 4.3 Assume that Condition (a) holds. The start-up's loss $W(y)$ due to incomplete information is given as follows.

Case (U): $\tilde{Q}_{2}<Q_{2}$ 
Case (U.1): $p\left(\beta_{1}, Q_{1}, \tilde{Q}_{2}\right) \leq 0 \quad W(y)=0$ for all $y>0$.

Case (U.2): $p\left(\beta_{1}, Q_{1}, \tilde{Q}_{2}\right)>0$ and $p\left(\beta_{1}, Q_{1}, Q_{2}\right) \leq 0 \quad W(y)=-\tilde{V}(y)$ for all $y>0$.

Case (U.3): $p\left(\beta_{1}, Q_{1}, Q_{2}\right)>0$

$$
W(y)= \begin{cases}0 & \left(0<y<y_{U}\left(Q_{2}\right)\right) \\ B\left(Q_{2}\right) y^{\beta_{2}}-\frac{D_{1} y}{\rho-\mu}+I_{1}+\frac{D_{1} \max \left(y, y_{M}\left(Q_{2}\right)\right)^{-\beta_{1}+1} y^{\beta_{1}}}{\rho-\mu} & \left(y_{U}\left(Q_{2}\right) \leq y \leq y_{U}\left(\tilde{Q}_{2}\right)\right) \\ \left(B\left(Q_{2}\right)-\tilde{B}\left(\tilde{Q}_{2}\right)\right) y^{\beta_{2}} & \left(y>y_{U}\left(\tilde{Q}_{2}\right)\right) .\end{cases}
$$

Case (C): $\tilde{Q}_{2}=Q_{2} \quad W(y)=0$ for all $y>0$.

Case $(\mathbf{O}): \tilde{Q}_{2}>Q_{2}$

Case (O.1): $p\left(\beta_{1}, Q_{1}, Q_{2}\right) \leq 0 \quad W(y)=0$ for all $y>0$.

Case (O.2): $p\left(\beta_{1}, Q_{1}, Q_{2}\right)>0$ and $p\left(\beta_{1}, Q_{1}, \tilde{Q}_{2}\right) \leq 0 \quad W(y)=V\left(y ; Q_{2}\right)$ for all $y>0$.

Case (O.3): $p\left(\beta_{1}, Q_{1}, \tilde{Q}_{2}\right)>0$

$$
W(y)= \begin{cases}0 & \left(0<y<y_{U}\left(\tilde{Q}_{2}\right)\right) \\ \frac{D_{1} y}{\rho-\mu}-I_{1}-\frac{D_{1} y_{M}\left(Q_{2}\right)^{-\beta_{1}+1} y^{\beta_{1}}}{\rho-\mu}-\tilde{B}\left(\tilde{Q}_{2}\right) & \left(y_{U}\left(\tilde{Q}_{2}\right) \leq y \leq y_{U}\left(Q_{2}\right)\right) \\ \left(B\left(Q_{2}\right)-\tilde{B}\left(\tilde{Q}_{2}\right)\right) y^{\beta_{2}} & \left(y>y_{U}\left(Q_{2}\right)\right) .\end{cases}
$$

Here, $y_{U}(\cdot)$ is the unique solution of equation $(13)$, and $B\left(Q_{2}\right)$ and $\tilde{B}\left(\tilde{Q}_{2}\right)$ are defined by (14) with $q=Q_{2}$ and (23), respectively.

(Proof) In Case $(\mathrm{C})$ (i.e., $\left.\tilde{Q}_{2}=Q_{2}\right)$, we have $\tilde{V}(y)=V\left(y ; Q_{2}\right)$ and hence $W(y)=$ $V\left(y ; Q_{2}\right)-\tilde{V}(y)=0$. By $(9)$, in Case (U) (i.e., $\left.\tilde{Q}_{2}<Q_{2}\right)$ we have $p\left(\beta_{1}, Q_{1}, Q_{2}\right)<$ $p\left(\beta_{1}, Q_{1}, \tilde{Q}_{2}\right)$, while in Case $(\mathrm{O})$ (i.e., $\left.\tilde{Q}_{2}>Q_{2}\right)$ we have $p\left(\beta_{1}, Q_{1}, \tilde{Q}_{2}\right)<p\left(\beta_{1}, Q_{1}, Q_{2}\right)$. Therefore, we can further classify Cases $(\mathrm{O})$ and $(\mathrm{U})$ into six regions. Then, we can easily calculate $W(y)=V\left(y ; Q_{2}\right)-\tilde{V}(y)$ from Propositions 3.1 and 4.2 in each case.

Let us mention how the start-up suffers the loss due to incomplete information in each case of the above proposition. Needless to say, in Case (C) the start-up's strategy becomes optimal as $\tau_{1}^{*}=\tau_{1}^{\tilde{Q_{2}}}=\tau_{1}^{Q_{2}}$, and hence the start-up suffers no loss for any initial value. In Cases (U.1) and (O.1), the prerequisite condition for the start-up's investment does not actually hold (i.e., $p\left(\beta_{1}, Q_{1}, Q_{2}\right) \leq 0$ ), and the start-up never attempts to invest. As a result, in these cases the start-up's never investment strategy is optimal, and the loss is always zero. 
Cases (U.2) and (O.2) correspond, respectively, to the case where the start-up attempts to invest although the prerequisite condition does not actually hold and the case where the start-up never attempts to invest although the prerequisite condition actually holds. Due to this misjudgment of the opportunity to invest, the start-up suffers the loss for any initial value $y>0$. Note that $W(y)$ in Case (U.2) is positive by $\tilde{V}(y)<0$ for all $y>0$.

In Cases (U.3) and (O.3), the prerequisite condition actually holds, and also the startup attempts to invest. Thus, in these cases, unlike in Cases (U.2) and (O.2), the start-up's judgement of the investment opportunity is correct. The start-up makes its investment at $\tau_{1}^{\tilde{Q_{2}}}=\inf \left\{t \geq 0 \mid Y(t) \in\left[y_{M}\left(Q_{1}\right), y_{U}\left(\tilde{Q}_{2}\right)\right]\right\}$, though the optimal investment timing $\tau_{1}^{Q_{2}}$ is given as $\inf \left\{t \geq 0 \mid Y(t) \in\left[y_{M}\left(Q_{1}\right), y_{U}\left(Q_{2}\right)\right]\right\}$. In Case (U.3), since $y_{U}\left(\tilde{Q}_{2}\right)>y_{U}\left(Q_{2}\right)$, the start-up makes the investment earlier than $\tau_{1}^{Q_{2}}$ and suffers the loss $W(y)$ when $y>y_{U}\left(Q_{2}\right)$; contrarily, in Case $(\mathrm{O} .3)$, since $y_{U}\left(\tilde{Q}_{2}\right)<y_{U}\left(Q_{2}\right)$, the start-up makes the investment later than $\tau_{1}^{Q_{2}}$ and suffers the loss $W(y)$ when $y>y_{U}\left(\tilde{Q}_{2}\right)$. The loss in the second region in Case (U.3) can be interpreted as the value of the option to defer the investment minus the value of the immediate investment. On the other hand, the loss in the second region in Case (O.3) represents the value of the immediate investment minus the value of the option to defer the investment

Corollary 4.1 Suppose that Condition (a) holds. Also assume that the random variable $X$ has a support $\left(0, Q_{U}\right]$ for some constant $Q_{U}$, and that the large firm's real efficiency $Q_{2}$ satisfies $Q_{2} \in\left(0, Q_{U}\right]$. If conditions $p\left(\beta_{1}, Q_{1}, Q_{U}\right)>0$ and $y \leq y_{U}\left(Q_{U}\right)$ are satisfied, then the start-up suffers no loss due to incomplete information. Here, $y_{U}\left(Q_{U}\right)$ is defined as the unique solution of equation (13) with $q=Q_{U}$.

The first condition means that it is certain that the efficiency of the start-up's investment, $Q_{1}$, is sufficiently greater than that of the large firm, $Q_{2}$. The second condition means that the initial market demand $Y(0)=y$ cannot generate great profit immediately. Thus, Proposition 4.3 suggests that more detailed information about the large firm is of little value when the start-up's efficiency is much better than that of the large firm in the new market that is small for the present.

The expected payoff $\tilde{V}(y)$ obtained by the real options strategy $\tau_{1}^{*}$ may generate less profit than the expected payoff $\tilde{V}_{N P V}(y)$ obtained by the zero-NPV strategy (which means to invest when the NPV of the investment becomes positive) under the same estimation 
$X$. To see this, consider the function $g(y)$ defined by (15) and assume that the equation $g(y)=0(y>0)$ has exactly two solutions denoted $0<y_{L}^{N P V}<y_{U}^{N P V}$ as shown in Figure 1. This assumption holds in most cases. Then, the start-up that employs the zero-NPV strategy invests at $\tau_{1}^{N P V}=\inf \left\{t \geq 0 \mid Y(t) \in\left[y_{L}^{N P V}, y_{U}^{N P V}\right]\right\}$, although the start-up that takes the real options strategy invests at $\tau_{1}^{*}=\inf \left\{t \geq 0 \mid Y(t) \in\left[y_{M}\left(Q_{1}\right), y_{U}\left(\tilde{Q}_{2}\right)\right]\right\}$. Since $y_{L}^{N P V}<y_{M}\left(Q_{1}\right)<y_{U}\left(\tilde{Q}_{2}\right)<y_{U}^{N P V}$ as observed in Figure 1, the zero-NPV timing $\tau_{1}^{N P V}$ is not later than the real options timing $\tau_{1}^{*}$. We define $Q_{N P V}$ as the unique solution of $y_{U}(q)=y_{U}^{N P V}$. Taking into consideration that the zero-NPV timing is expressed as $\inf \left\{t \geq 0 \mid Y(t) \in\left[y_{L}^{N P V}, y_{U}\left(Q_{N P V}\right)\right]\right\}$, we can show the following corollary.

Corollary 4.2 Suppose that Condition (a) holds. Also assume that the equation $g(y)=$ $0(y>0)$ has exactly two solutions. Then, $\tilde{V}_{N P V}(y)>\tilde{V}(y)$ holds if one of the following three conditions is satisfied in Case (O.3) (i.e., $\tilde{Q}_{2}>Q_{2}$ and $\left.p\left(\beta_{1}, Q_{1}, \tilde{Q}_{2}\right)>0\right)$ :

- $Q_{N P V}<Q_{2}$ and $y>y_{U}\left(\tilde{Q}_{2}\right)$

- $Q_{2} \leq Q_{N P V}, \tilde{B}\left(\tilde{Q}_{2}\right)<\tilde{B}\left(Q_{N P V}\right)$ and $y>y_{U}\left(\tilde{Q}_{2}\right)$

- $Q_{2} \leq Q_{N P V}, \tilde{B}\left(Q_{N P V}\right) \leq \tilde{B}\left(\tilde{Q}_{2}\right)$ and $y_{U}\left(\tilde{Q}_{2}\right)<y<y_{C}$

Here, $y_{U}\left(\tilde{Q}_{2}\right)$ is the unique solution of equation (13) with $q=\tilde{Q}_{2}$ and $\tilde{B}(\cdot)$ is defined by (23). Moreover, $y_{C}$ is the unique solution of the equation

$\frac{D_{1}}{\rho-\mu} \max \left(y, y_{M}\left(Q_{2}\right)\right)^{-\beta_{1}+1} y^{\beta_{1}}+\tilde{B}\left(\tilde{Q}_{2}\right) y^{\beta_{2}}-\frac{D_{1}}{\rho-\mu} y+I_{1}=0 \quad\left(y_{U}\left(Q_{2}\right)<y \leq y_{U}\left(Q_{N P V}\right)\right)$, which is obtained as the intersection of the graphs of two functions $\tilde{V}_{N P V}(y)=D_{1} y /(\rho-$ $\mu)-I_{1}-D_{1} \max \left(y, y_{M}\left(Q_{2}\right)\right)^{-\beta_{1}+1} y^{\beta_{1}} /(\rho-\mu)$ and $\tilde{V}(y)=\tilde{B}\left(\tilde{Q}_{2}\right) y^{\beta_{2}}$.

\section{General setting}

This section makes a brief explanation about results in the general situation where the large follower does not completely annihilate the small firm, i.e., we assume that $0<$ $D_{1}(1,1)<D_{1}(1,0)$. In practice, a small entrepreneurial firm (or its unique technology) may tend to be bought-out by a large follower. The analysis in this section could be useful in such a situation by reinterpreting the expected profit $E\left[\int_{\tau_{2}}^{+\infty} \mathrm{e}^{-\rho t} D_{1}(1,1) Y(t) \mathrm{d} t\right]$ after the large firm's entry time $\tau_{2}$ as the reward which the start-up gains by the buy-out. 
The difference from the results of the previous sections only consists in the fact that the start-up's investment policy involves one more investment trigger. First, let us consider the case of complete information. The start-up's investment strategy can be written as $\tau_{1}^{q}=\inf \left\{t \geq 0 \mid Y(t) \in\left[y_{M}\left(Q_{1}\right), y_{U_{1}}(q)\right] \cup\left[y_{U_{2}}(q),+\infty\right)\right\}$ under a similar condition (but much more complicated since it involves $D_{1}(1,1)$ in addition to $\beta_{1}, Q_{1}$, and $\left.q\right)$ to the prerequisite condition in Proposition 3.1. The additional stopping (investment) region $\left[y_{U_{2}}(q),+\infty\right)$ represents the start-up's investment allowing the large firm's immediate follow. Indeed, for any sufficiently large market demand $Y(t)$, the start-up obtains the positive profit $D(1,1) Y(t) /(\rho-\mu)-I_{1}$ (note $D_{1}(1,1)>0$ ) in spite of the large firm's immediate follow. Note that this region is not important in terms of clarifying the feature of the small firm's strategy as the leader. The start-up does not have to wait forever for large $Y(t)$, though for halfway $Y(t) \in\left(y_{U_{1}}(q), y_{U_{2}}(q)\right)$, it delays the investment until $Y(t)$ either falls to $y_{U_{1}}(q)$ or rises to $y_{U_{2}}(q)$. The condition (called, hereafter, the preemptive condition), which is obtained by modifying the prerequisite condition, determines whether the start-up takes a preemptive action. Recall that the prerequisite condition determines whether the start-up completely gives up. If the preemptive condition is not satisfied, the start-up's strategy can be expressed as $\left.\tau_{1}^{q}=\inf \left\{t \geq 0 \mid Y(t) \geq y_{M}\left(D_{1}(1,1) / I_{1}\right)\right)\right\}$. In this case, the start-up gives up any entrepreneurial action, instead of completely giving up the investment.

Next, we consider the case of incomplete information. Similar results to those in Sections 4.1 and 4.2 are obtained by modifying the definition of $g(y)$. With the definition of $\tilde{Q}_{2}$ unchanged, we can still classify the start-up's strategy into Cases (U), (C), and $(\mathrm{O})$, according to the relation between $Q_{2}$ and $\tilde{Q}_{2}$. Therefore, the essence of the results about the start-up's loss due to incomplete information is preserved. Indeed, the results differ from those in Section 4.3 only in that the loss $W(y)$ always becomes zero for a sufficiently large initial value $y$ because the start-up's optimal investment strategy allows the large firm's immediate follow. There is little difference between the results in the previous sections and those of the general situation when the initial value $y$ is small, and therefore the same statement as in Corollary 4.1 holds. 


\section{$6 \quad$ Numerical Examples}

This section shows economic implications, using some numerical examples, of the theoretical results given in Sections 3 and 4. Unless otherwise noted, in what follows we set the start-up's parameters as $D_{1}=0.04, I_{1}=1, \mu=0, \sigma=0.2$, and $\rho=0.04$ as in the standard parameter values in Dixit and Pindyck (1994). Then, we have $Q_{1}=0.04, \beta_{1}=2$ and $\beta_{2}=-1$. Moreover, the investment trigger for the monopolist is calculated as $y_{M}\left(Q_{1}=0.04\right)=2$, which is twice as big as the Marshallian trigger (i.e., the point where the NPV is zero).

To begin with, we consider the start-up's investment strategy with complete information for a range of the large firm's efficiency $Q_{2}=q$. We observe from Figure 2 that, if the large firm's efficiency $Q_{2}=q$ is less than half of the start-up's efficiency $Q_{1}=0.04$, the prerequisite condition (10) for the start-up's investment is satisfied. The vertical dotted line at $q=0.02$ in Figure 2 divides the whole region into two subregions that correspond to the case where the prerequisite condition (10) holds and the case of never investing. The former subregion is further divided into three regions as shown in Figure 2. We can observe that the start-up's investment region becomes larger as the large firm's efficiency $Q_{2}=q$ decreases.

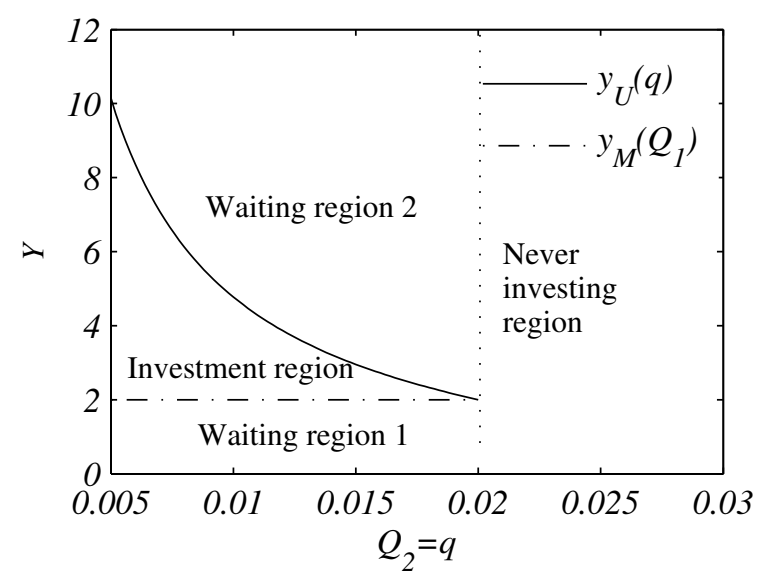

Figure 2: Investment triggers for various $Q_{2}=q$.

Let us turn our attention to the strategy and the loss $W(y)$ of the start-up with estimations $X$ of the large firm's efficiency. We examine two different cases: (1) the prerequisite condition (10) is really satisfied, in which case we set the large firm's real 
efficiency $Q_{2}=0.01$, and (2) the prerequisite condition (10) does not hold, in which case we set $Q_{2}=0.03$. Tables 1 and 2 show the resulting values in cases (1) and (2), respectively. The second column in the tables represents the resulting cases defined in Proposition 4.3. The notation $\emptyset$ in the third column means that the start-up never invests regardless of the value of the market demand $Y(t)$. Note that $\tilde{Q}_{2}=E[X]$ because of $\beta_{1}=2$. In addition, Condition (a) given in Subsection 4.1 is always satisfied in the examples. The tables show the losses $W(y)$ for different initial values $y=1,4$ and 10 . Note that, for $Q_{2}=0.01, y=1,4$ and 10 lie in the waiting region 1 , the investment region, and the waiting region 2 in Figure 2, respectively.

In Table 1, the losses $W(1)$ and $W(4)$ are zero for several distributions of $X$ in spite of the misestimation. This corresponds to the fact that for a small initial market demand no loss occurs, which is shown in Proposition 4.3 and Corollary 4.1. For a large initial market demand, comparing $W(10)=1.55,0.41$ and 0.23,0.097 in Table 1 suggests that the loss in the overestimation case (O.3) is smaller than that in the underestimation case (U.3). Taking into account that the value in the case of complete information is $V(10,0.01)=0.55$, even a small estimation error (in particular, in the underestimation case) causes a serious problem to the start-up. Indeed, the losses $W(10)=1.55$ and 0.41 correspond to 280 and 75 percents of $V(10,0.01)=0.55$, respectively. In the case where the prerequisite condition does not hold, on the other hand, a small estimation error causes no problem to the start-up. This can be seen from $W(y)=0$ in many rows above and below $X=Q_{2}=0.03$ in Table 2 . In fact, the start-up can make a correct judgement of never investing even if it has a small estimation error.

From the above observation, we obtain the following implications about the startup's investment policy under incomplete information. The start-up needs more accurate estimation of the large firm's efficiency in the case where it tries to invest than in the case of never investing. In addition, the start-up that attempts to invest should make a modest estimate because the loss in the underestimation case is likely to be much larger than that in the overestimation case. The start-up's strategy in the underestimation case may cause a loss larger than the project value in the case of complete information, and therefore its confident investment policy is excessively risky.

Finally, we show interesting numerical comparative static results with respect to the 
volatility $\sigma$ in the underlying market demand $Y(t)$. We set the large firm's efficiency $Q_{2}=0.01$. Figure 3 illustrates the start-up's value function $V\left(y ; Q_{2}=0.01\right)$ in the case of complete information for $\sigma=0,0.1,0.2$ and 0.3 . For an initial value around the investment region (approximately $[1.5,4.5]$ in Figure 3) a lower volatility generates a higher value of the start-up's investment, while for an initial value in the waiting regions (especially, the waiting region 2 such as $y \approx 10$ ) a higher volatility is beneficial to the start-up.

This result is intuitive. Note that the possibilities of both firm's entries in the waiting regions become smaller as the volatility in the market becomes lower. In other words, the value of the option to delay the investment is monotonic with respect to the volatility in the market. Then, a lower volatility has both positive and negative effects on the start-up. The positive one is that a lower volatility leads the large firm to delay its entry. The negative one is that a lower volatility decreases the start-up's option value of waiting. Since around the investment region the start-up can invest soon even if the volatility in the market is small, the negative effect in the start-up's waiting region is not important. As a result, around the investment region, a lower volatility increases the start-up's value by the positive effect. Far from the investment region, on the other hand, the negative effect is dominant, because the start-up tends to wait for a long time until its investment. In consequence, far from the investment region, a lower volatility decreases the start-up's value by the negative effect.

Figure 4 illustrates the relative loss $W(y) / V\left(y ; Q_{2}\right)$ of the start-up with incomplete information $X=[0.005,0.01]$ and $[0.01,0.015]$ for various values of $\sigma$. As shown in Proposition 4.3 and Corollary 4.1, the relative loss is zero for a small initial value $y$ in Figure 4. Observe that, for a large initial value $y$, the relative loss is constant with respect to $y$. This is because $W(y) / V\left(y ; Q_{2}\right)$ equals $\left(B\left(Q_{2}\right)-\tilde{B}\left(\tilde{Q}_{2}\right)\right) y^{\beta_{2}} / B\left(Q_{2}\right) y^{\beta_{2}}=$ $\left(B\left(Q_{2}\right)-\tilde{B}\left(\tilde{Q}_{2}\right)\right) / B\left(Q_{2}\right)$ by Proposition 4.3. In Figure 4, a lower volatility increases both the relative loss $W(y) / V\left(y ; Q_{2}\right)$ and the absolute loss $W(y)$. We observed that the same property holds for most other parameter values than the presented example.

We can interpret this property as follows. The start-up's investment decision involves two different types of uncertainty; namely the market volatility and the estimation of the large firm's efficiency. Intuitively, in the market with high volatility, a small estimation 
error does not make a big difference in the loss. However, if the uncertainty in the market demand is less, the start-up's payoff is more decisively determined by its investment policy. Naturally, the start-up also needs to take a more accurate investment policy.

Table 1: The loss for uniform distributions $X$ and $Q_{2}=0.01$.

\begin{tabular}{cccccc}
\hline$X$ & Case & Investment region & $W(1)$ & $W(4)$ & $W(10)$ \\
\hline$[0.0025,0.0075]$ & $(\mathrm{U} .3)$ & {$[2,10.14]$} & 0 & 0 & 1.55 \\
{$[0.005,0.01]$} & $(\mathrm{U} .3)$ & {$[2,6.57]$} & 0 & 0 & 0.41 \\
$Q_{2}=0.01$ & $(\mathrm{C})$ & {$[2,4.77]$} & 0 & 0 & 0 \\
{$[0.01,0.015]$} & $(\mathrm{O} .3)$ & {$[2,3.69]$} & 0 & 0.089 & 0.097 \\
{$[0.0125,0.0175]$} & $(\mathrm{O} .3)$ & {$[2,2.95]$} & 0 & 0.36 & 0.23 \\
{$[0.015,0.02]$} & $(\mathrm{O} .3)$ & {$[2,2.42]$} & 0 & 0.58 & 0.34 \\
{$[0.0175,0.0225]$} & $(\mathrm{O} .2)$ & $\emptyset$ & 0.13 & 1 & 0.55 \\
{$[0.02,0.025]$} & $(\mathrm{O} .2)$ & $\emptyset$ & 0.13 & 1 & 0.55 \\
\hline
\end{tabular}

Table 2: The loss for uniform distributions $X$ and $Q_{2}=0.03$.

\begin{tabular}{cccccc}
\hline$X$ & Case & Investment region & $W(1)$ & $W(4)$ & $W(10)$ \\
\hline$[0.0125,0.0175]$ & $(\mathrm{U} .2)$ & {$[2,2.95]$} & 0.13 & 0.74 & 0.37 \\
{$[0.015,0.02]$} & $(\mathrm{U} .2)$ & {$[2,2.42]$} & 0.13 & 0.47 & 0.23 \\
{$[0.0175,0.0225]$} & $(\mathrm{U} .1)$ & $\emptyset$ & 0 & 0 & 0 \\
{$[0.02,0.025]$} & $(\mathrm{U} .1)$ & $\emptyset$ & 0 & 0 & 0 \\
{$[0.0225,0.0275]$} & $(\mathrm{U} .1)$ & $\emptyset$ & 0 & 0 & 0 \\
{$[0.025,0.03]$} & $(\mathrm{U} .1)$ & $\emptyset$ & 0 & 0 & 0 \\
$Q_{2}=0.03$ & $(\mathrm{C})$ & $\emptyset$ & 0 & 0 & 0 \\
{$[0.03,0.035]$} & $(\mathrm{O} .1)$ & $\emptyset$ & 0 & 0 & 0 \\
{$[0.0325,0.0375]$} & $(\mathrm{O} .1)$ & $\emptyset$ & 0 & 0 & 0 \\
\hline
\end{tabular}




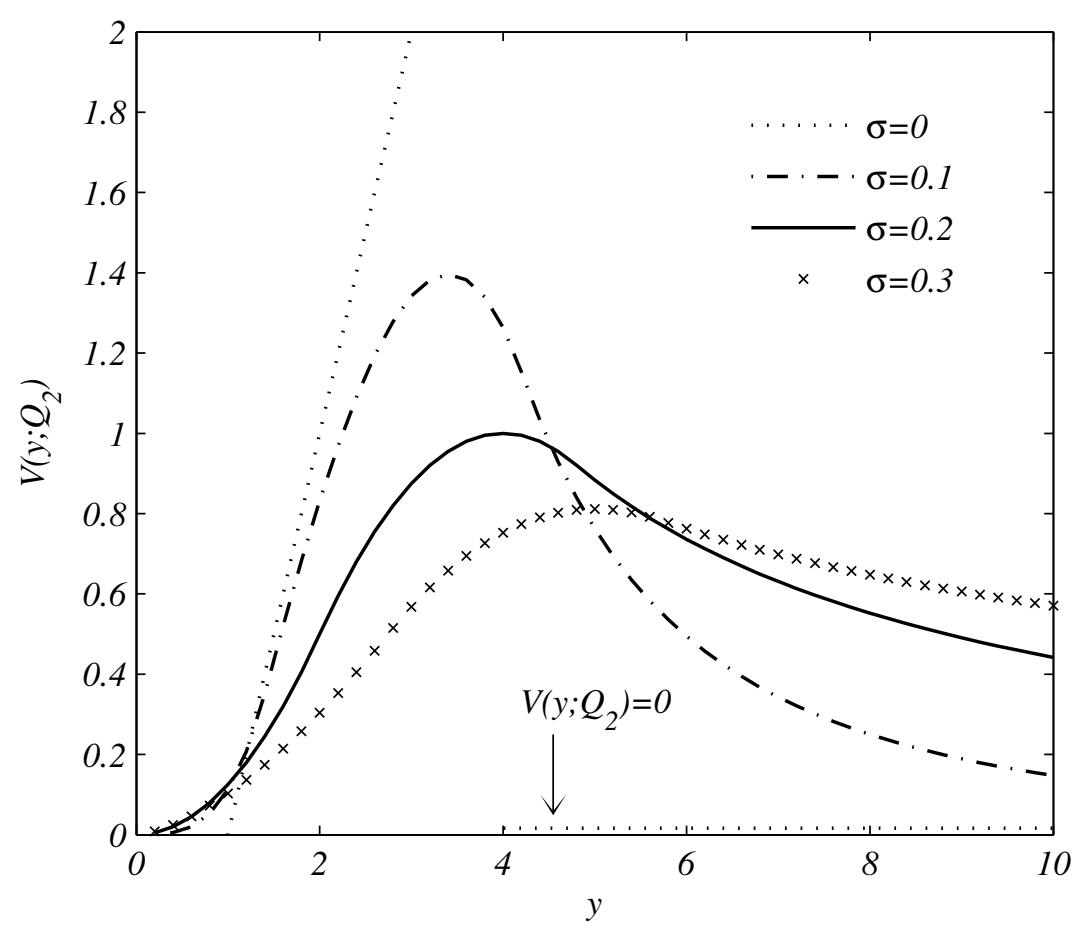

Figure 3: $V\left(y ; Q_{2}=0.01\right)$ for $\sigma=0,0.1,0.2$ and 0.3 .

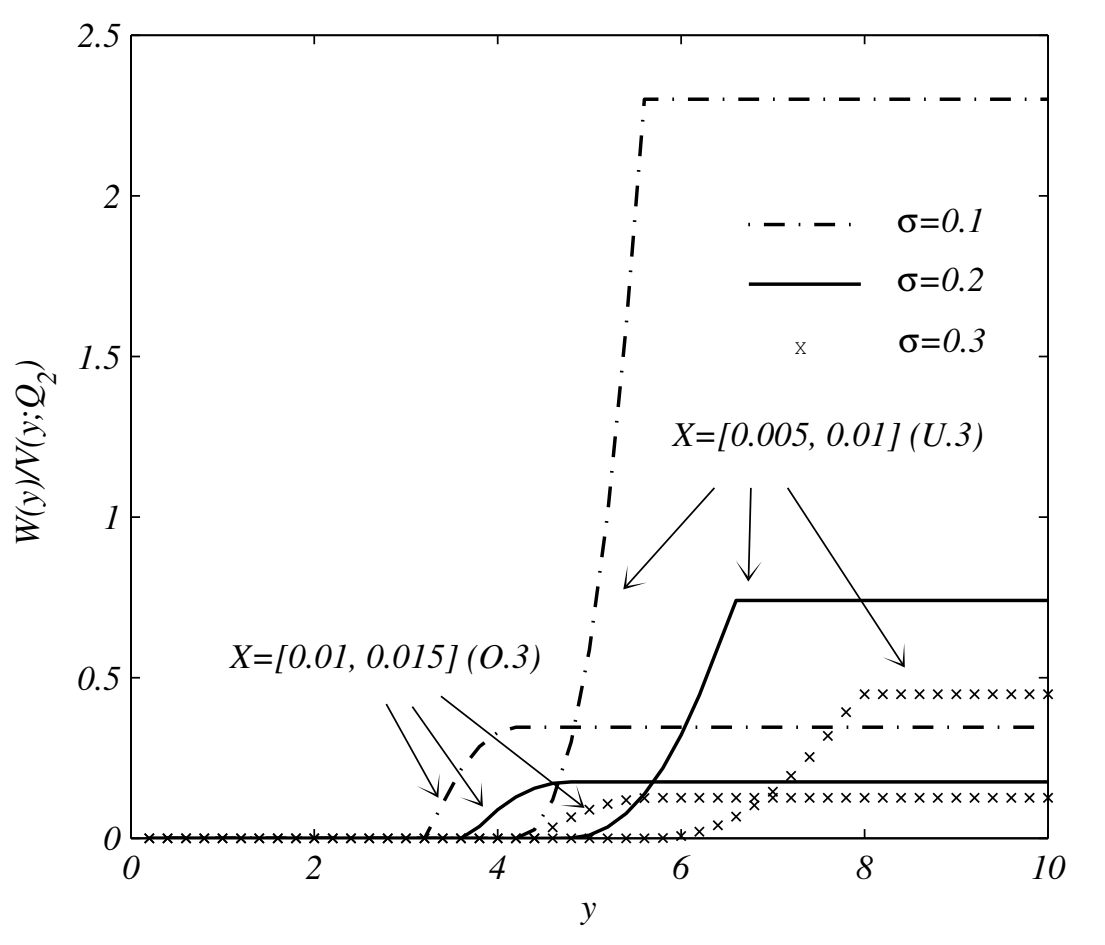

Figure 4: $W(y) / V\left(y ; Q_{2}=0.01\right)$ for $\sigma=0.1,0.2$ and 0.3 . 


\section{Conclusion}

This paper has investigated the effect of incomplete information in the model in which a start-up with a unique idea and technology pioneers a new market that will be taken over by a large firm eventually. The main contribution of this paper is to evaluate the start-up's loss due to incomplete information about the large firm. The proposed method could be applied in other real options models involving several firms. The results obtained in this paper can be summarized as follows.

If the start-up's efficiency is much better than that of the large firm and the current market demand cannot generate great profit immediately, then the start-up requires no further survey on the large firm's efficiency. On the other hand, information about the large firm's efficiency is valuable in the market that can readily generate great profit, even if the start-up's efficiency is much better than that of the large firm. In this case, it is quite likely that the start-up's immediate investment does not produce much income for the start-up before the large firm's entry.

When it is doubtful that the start-up's efficiency overwhelms that of the large firm, information about the large firm's efficiency is always valuable regardless of the current market demand. The reason for this is that there is a possibility that the investment opportunity for the start-up does not exist in the market, in addition to the same risk as in the previous case, that is, the possibility that the start-up obtains little profit before the large firm's entry.

Furthermore, under incomplete information, the expected payoff of the start-up investing at the zero-NPV trigger could become greater than that of the start-up following the real options approach. In numerical examples, we have also observed some interesting features of the loss due to incomplete information such as the property that the loss in the overestimation case tends to be smaller than that in the underestimation case.

In the real world, a small entrepreneurial firm that has a unique idea and technology but is not competitive in the market may want to sell its idea and technology to a large firm, rather than pioneering the market by itself. Then, the value function which the start-up believes can be interpreted as a reward which the start-up demands for its idea and technology. As revealed in this paper, the value of the investment which the start-up believes under incomplete information is generally different from the real value of the 
investment. Because of this gap, negotiations between the start-up and the large firm may not go smoothly. It remains as an interesting issue of future research to reveal the effect of incomplete information in such a negotiation problem of a firm having an option to sell its idea and technology to the rival firm.

\section{Appendix A Proof of Proposition 3.1}

Taking account of (5), we can compute (3) as follows:

$$
\begin{aligned}
& E\left[\int_{\tau_{1}}^{\tau_{2}^{q}} \mathrm{e}^{-\rho t} D_{1} Y(t) \mathrm{d} t-\mathrm{e}^{-\rho \tau_{1}} I_{1}\right] \\
= & E\left[\mathrm{e}^{-\rho \tau_{1}}\left(D_{1} E^{Y\left(\tau_{1}\right)}\left[\int_{0}^{\tau_{2}^{q}} \mathrm{e}^{-\rho t} Y(t) \mathrm{d} t\right]-I_{1}\right)\right] \\
= & E\left[\mathrm{e}^{-\rho \tau_{1}}\left(D_{1} E^{Y\left(\tau_{1}\right)}\left[\int_{0}^{+\infty} \mathrm{e}^{-\rho t} Y(t) \mathrm{d} t-\int_{\tau_{2}^{q}}^{+\infty} \mathrm{e}^{-\rho t} Y(t) \mathrm{d} t\right]-I_{1}\right)\right] \\
= & E\left[\mathrm{e}^{-\rho \tau_{1}}\left(D_{1} E^{Y\left(\tau_{1}\right)}\left[\int_{0}^{+\infty} \mathrm{e}^{-\rho t} Y(t) \mathrm{d} t-\mathrm{e}^{-\rho \tau_{2}^{q}} E^{Y\left(\tau_{2}^{q}\right)}\left[\int_{0}^{+\infty} \mathrm{e}^{-\rho t} Y(t) \mathrm{d} t\right]\right]-I_{1}\right)(25)\right. \\
= & E\left[\mathrm{e}^{-\rho \tau_{1}}\left(D_{1} E^{Y\left(\tau_{1}\right)}\left[\int_{0}^{+\infty} \mathrm{e}^{-\rho t} Y(t) \mathrm{d} t-\frac{\mathrm{e}^{-\rho \tau_{2}^{q}} \max \left(Y(0), y_{M}(q)\right)}{\rho-\mu}\right]-I_{1}\right)\right] \\
= & E\left[\mathrm{e}^{-\rho \tau_{1}}\left(\frac{D_{1} Y\left(\tau_{1}\right)}{\rho-\mu}-\frac{D_{1} \max \left(Y\left(\tau_{1}\right), y_{M}(q)\right)^{-\beta_{1}+1} Y\left(\tau_{1}\right)^{\beta_{1}}}{\rho-\mu}-I_{1}\right)\right]
\end{aligned}
$$

where we use the strong Markov property (e.g. see Øksendal (2003)) of the geometric Brownian motion $Y(t)$ to deduce (24) and (25), and use the formula of the expectation involving a hitting time (e.g. see Dixit and Pindyck (1994)) to deduce (26). Here, for a random variable $Z, E^{Y\left(\tau_{i}\right)}[Z]$ denotes a random variable $G\left(Y\left(\tau_{i}\right)\right)$, where for $y^{\prime}>0$, $G\left(y^{\prime}\right)$ is defined as an expectation $E[Z]$ in the case where $Y(t)$ starts at $Y(0)=y^{\prime}$. Thus, problem (4) with $X \equiv q$ is equivalent to $\sup _{\tau_{1}} E\left[\mathrm{e}^{-\rho \tau_{1}} f\left(Y\left(\tau_{1}\right) ; q\right)\right]$, where

$$
f(y ; q)=\frac{D_{1} y}{\rho-\mu}-\frac{D_{1} \max \left(y, y_{M}(q)\right)^{-\beta_{1}+1} y^{\beta_{1}}}{\rho-\mu}-I_{1} .
$$

Consider the case where $f(y ; q) \leq 0$ for all $y>0$. In this case, the value function and the optimal stopping time are trivially given by $V(y ; q)=0$ and $\tau_{1}^{q}=+\infty$, respectively, for all $y>0$. Now, let us derive a necessary and sufficient condition for $f(y ; q) \leq 0$ to hold for all $y>0$. Since $f(y ; q)$ is concave for $y \in\left[0, y_{M}(q)\right]$ by $\beta_{1}>1$ and $f(y ; q)=-I_{1}$ holds for $y=0$ and $y \geq y_{M}(q), f(y ; q)(y>0)$ takes the maximum value at $y=$ 
$\beta_{1}^{-1 /\left(\beta_{1}-1\right)} y_{M}(q)$, which is the unique solution of $\partial f(y ; q) / \partial y=0$ for $y \in\left[0, y_{M}(q)\right]$. Since we have $f\left(\beta_{1}^{-1 /\left(\beta_{1}-1\right)} y_{M}(q) ; q\right)=D_{1} p\left(\beta_{1}, Q_{1}, q\right) / q$ by (6), (9) and (27), we can deduce that $p\left(\beta_{1}, Q_{1}, q\right) \leq 0$ is a necessary and sufficient condition for $f(y ; q) \leq 0$ to hold for all $y>0$. Thus, if $p\left(\beta_{1}, Q_{1}, q\right) \leq 0$, we have $V(y ; q)=0$ and $\tau_{1}^{q}=+\infty$.

Next, we consider the case where $p\left(\beta_{1}, Q_{1}, q\right)>0$. In this case, if we can check that the right-hand side of $(11)$, denoted $\phi(y)$, is a continuously differentiable function satisfying the following conditions:

$$
\begin{aligned}
& \frac{\sigma^{2} y^{2}}{2} \frac{\mathrm{d}^{2} \phi}{\mathrm{d} y^{2}}(y)+\mu y \frac{\mathrm{d} \phi}{\mathrm{d} y}(y)-\rho \phi(y)\left\{\begin{array}{l}
\leq 0 \text { for all } y>0, \\
=0 \text { for all } y \notin\left[y_{M}\left(Q_{1}\right), y_{U}(q)\right],
\end{array}\right. \\
& \phi(y)-f(y)\left\{\begin{array}{l}
\geq 0 \text { for all } y>0, \\
=0 \text { for all } y \in\left[y_{M}\left(Q_{1}\right), y_{U}(q)\right],
\end{array}\right. \\
& \lim _{y \downarrow 0} \phi(y)=\lim _{y \uparrow+\infty} \phi(y)=0, \\
& \phi(y): \text { twice continuously differentiable at any } y \neq y_{M}\left(Q_{1}\right), y_{U}(q),
\end{aligned}
$$

then we obtain the value function $V(y ; q)=\phi(y)$ and the optimal stopping time $\tau_{1}^{q}=$ $\inf \left\{t \geq 0 \mid Y(t) \in\left[y_{M}\left(Q_{1}\right), y_{U}(q)\right]\right\}$ via the relation between optimal stopping and variational inequalities (for details see Øksendal (2003)). Note that the thresholds $y_{M}(q)$ and $y_{U}(q)$ are defined so that $\phi(y)$ is continuously differentiable at the thresholds (i.e, value matching and smooth pasting, see also Dixit and Pindyck (1994)). Since we can check all the conditions for $\phi(y)$ by direct calculation, we obtain the proposition.

\section{Appendix B Proof of Proposition 4.1}

Note that

$$
\begin{aligned}
& E\left[\int_{\tau_{1}}^{\tau_{2}^{X}} \mathrm{e}^{-\rho t} D_{1} Y(t) \mathrm{d} t+\int_{\tau_{2}^{X}}^{+\infty} \mathrm{e}-\mathrm{e}^{-\rho \tau_{1}} I_{1}\right] \\
= & \int_{0}^{+\infty} E\left[\int_{\tau_{1}}^{\tau_{2}^{X}} \mathrm{e}^{-\rho t} D_{1} Y(t) \mathrm{d} t-\mathrm{e}^{-\rho \tau_{1}} I_{1} \mid X=q\right] \mathrm{d} \Psi_{X}(q) \\
= & \int_{0}^{+\infty} E\left[\int_{\tau_{1}}^{\tau_{2}^{q}} \mathrm{e}^{-\rho t} D_{1} Y(t) \mathrm{d} t-\mathrm{e}^{-\rho \tau_{1}} I_{1}\right] \mathrm{d} \Psi_{X}(q) \\
= & \int_{0}^{+\infty} E\left[\mathrm{e}^{-\rho \tau_{1}} f\left(Y\left(\tau_{1}\right) ; q\right)\right] \mathrm{d} \Psi_{X}(q) \\
= & E\left[\mathrm{e}^{-\rho \tau_{1}} g\left(Y\left(\tau_{1}\right)\right)\right],
\end{aligned}
$$


where $\Psi_{X}(q)$ denotes the distribution of $X$, and $f$ and $g$ are defined by (27) and (15), respectively. Here, (29) and (31) follow from the independence between $X$ and $Y(t)$, and (30) follows from the strong Markov property as in Appendix A.

First, we consider the case where $g(y) \leq 0$ for all $y>0$. In this case, apparently, the value function and the optimal stopping time are given by $V(y)=0$ and $\tau_{1}^{*}=+\infty$, respectively, for all $y>0$. Since $h(y) \leq 0$ holds for all $y>0$ by $(17), V\left(y ; \tilde{Q}_{2}\right)=0$ and $\tau_{1}^{\tilde{Q_{2}}}=+\infty$ hold for all $y>0$. This implies $V(y)=V\left(y ; \tilde{Q}_{2}\right)$ and $\tau_{1}^{*}=\tau_{1}^{\tilde{Q}_{2}}$ for all $y>0$.

Next, let us assume that there exists some $\hat{y}>0$ such that $g(\hat{y})>0$. We have $V\left(\hat{y} ; \tilde{Q}_{2}\right)>0$ by Condition (a) (i.e., $g(y) \leq V\left(y ; \tilde{Q}_{2}\right)$ for all $\left.y>0\right)$. Then, we can deduce that $p\left(y, Q_{1}, \tilde{Q}_{2}\right)>0$, taking into consideration that $V\left(y ; \tilde{Q}_{2}\right)=0$ holds for all $y>0$ whenever $p\left(y, Q_{1}, \tilde{Q}_{2}\right) \leq 0$ by Proposition 3.1. We have only to check the conditions (28) with $q$ and $f$ replaced by $\tilde{Q}_{2}$ and $g$ for $\phi(y)=V\left(y ; \tilde{Q}_{2}\right)$ (i.e., the right-hand side of $(11)$ with $q$ replaced by $\tilde{Q}_{2}$ ). The conditions (28) except for the second can be checked directly as in Appendix A. Condition (a) ensures $\phi(y)-g(y) \geq 0$ for all $y>0$. By (17) and (18), for all $y \in\left[y_{M}\left(Q_{1}\right), y_{U}\left(\tilde{Q}_{2}\right)\right]$, we have $\phi(y)-g(y)=h(y)-g(y) \leq 0$, where $h(y)$ is defined by (16). These imply the second condition. Therefore, we obtain $V(y)=\phi(y)$ and $\tau_{1}^{*}=\inf \left\{t \geq 0 \mid Y(t) \in\left[y_{M}\left(Q_{1}\right), y_{U}\left(\tilde{Q}_{2}\right)\right]\right\}$ via the relation between optimal stopping and variational inequalities (e.g., see Øksendal (2003)).

\section{Appendix C $\quad$ Proof of Proposition 4.2}

We have only to compute the expectation (20). First, we assume $p\left(\beta_{1}, Q_{1}, \tilde{Q}_{2}\right) \leq 0$. In this case, we have $\tau_{1}^{*}=\tau_{1}^{\tilde{Q_{2}}}=+\infty$ by Propositions 3.1 and 4.1 , and hence we have also $\tau_{2}^{Q_{2}}=+\infty$ by (19). Thus, $\tilde{V}(y)=0$ holds for all $y>0$. Next, let us assume $p\left(\beta_{1}, Q_{1}, \tilde{Q}_{2}\right)>0$. In this case, we have

$$
\tau_{1}^{*}=\tau_{1}^{Q_{2}}=\inf \left\{t \geq 0 \mid Y(t) \in\left[y_{M}\left(Q_{1}\right), y_{U}\left(\tilde{Q}_{2}\right)\right]\right\}
$$

by Propositions 3.1 and 4.1. As in Appendix A, by the strong Markov property, (20) is equal to (26) with $\tau_{1}$ and $q$ replaced by $\tau_{1}^{*}$ and $Q_{2}$, respectively. That is, we have $\tilde{V}(y)=E\left[\mathrm{e}^{-\rho \tau_{1}^{*}} f\left(Y\left(\tau_{1}^{*}\right) ; Q_{2}\right)\right]$, where $f$ is defined by $(27)$. Since $Y\left(\tau_{1}^{*}\right)$ is a constant such 
that

$$
Y\left(\tau_{1}^{*}\right)= \begin{cases}y_{M}\left(Q_{1}\right) & \left(0<y<y_{M}\left(Q_{1}\right)\right) \\ y & \left(y_{M}\left(Q_{1}\right) \leq y \leq y_{U}\left(\tilde{Q}_{2}\right)\right) \\ y_{U}\left(\tilde{Q}_{2}\right) & \left(y>y_{U}\left(\tilde{Q}_{2}\right)\right)\end{cases}
$$

by (32), we have

$$
\tilde{V}(y)=f\left(Y\left(\tau_{1}^{*}\right) ; Q_{2}\right) E\left[\mathrm{e}^{-\rho \tau_{1}^{*}}\right]
$$

Thus, by applying the formula of the expectation involving a hitting time to (34), we obtain the formula of $\tilde{V}(y)$ given in the proposition.

\section{Acknowledgements}

The authors would like to thank an anonymous referee and the members of Professor Masaaki Kijima's Laboratory of Kyoto University for helpful suggestions. This research was partially supported by a MEXT Grant-in-Aid for the 21st Century COE Program "Informatics Research Center for Development of Knowledge Society Infrastructure" in Kyoto University.

\section{References}

Bernardo, A., and B. Chowdhry, 2002, Resources, real options, and corporate strategy, Journal of Financial Economics 63, 211-234.

Boyer, M., É. Gravel, and P. Lasserre, 2004, Real options and strategic competition: a survey, Working paper, Université de Montréal.

Décamps, J., T. Mariotti, and S. Villeneuve, 2005, Investment timing under incomplete information, Mathematics of Operations Research 30, 472-500.

Dixit, A., 1989, Entry and exit decisions under uncertainty, Journal of Political Economy $97,620-638$.

and R. Pindyck, 1994, Investment Under Uncertainty (Princeton University Press: Princeton). 
Grenadier, S., 2002, Option exercise games: an application to the equilibrium investment strategies of firms, Review of Financial Studies 15, 691-721.

— , and N. Wang, 2005, Investment timing, agency, and information, Journal of Financial Economics 75, 493-533.

Hsu, Y., and B. Lambrecht, 2003, Preemptive patenting under uncertainty and asymmetric information, Working paper.

Huisman, K., 2001, Technology Investment: A Game Theoretic Real Options Approach (Kluwer Academic Publishers: Boston).

, and P. Kort, 2003, Strategic investment in technological innovations, European Journal of Operational Research 144, 209-223.

Lambrecht, B., and W. Perraudin, 2003, Real options and preemption under incomplete information, Journal of Economics Dynamics and Control 27, 619-643.

McDonald, R., and D. Siegel, 1986, The value of waiting to invest, Quarterly Journal of Economics 101, 707-727.

Nishihara, M., and T. Shibata, 2007, The effects of auditing in a real options model, Technical Report 2007-1, Department of Applied Mathematics and Physics, Graduate School of Informatics, Kyoto University.

Øksendal, B., 2003, Stochastic Differential Equations: An Introduction with Applications 6th edition (Springer: Berlin).

Weeds, H., 2002, Strategic delay in a real options model of R\&D competition, Review of Economic Studies 69, 729-747. 